\title{
Chronique des travaux universitaires
}

Jean-Pierre Watté

\section{OpenEdition}

Journals

Édition électronique

URL : http://journals.openedition.org/rao/1398

DOI : $10.4000 /$ rao. 1398

ISSN : 1775-3732

\section{Éditeur}

Presses universitaires de Rennes

\section{Édition imprimée}

Date de publication : 31 décembre 2010

Pagination : 217-219

ISBN : 978-2-7535-1383-9

ISSN : 0767-709X

\section{Référence électronique}

Jean-Pierre Watté, "Chronique des travaux universitaires ", Revue archéologique de l'Ouest [En ligne] 27 | 2010, mis en ligne le 25 février 2012, consulté le 05 décembre 2020. URL : http:// journals.openedition.org/rao/1398; DOI : https://doi.org/10.4000/rao.1398

Ce document a été généré automatiquement le 5 décembre 2020

Tous droits réservés 


\title{
Chronique des travaux universitaires
}

\author{
Jean-Pierre Watté
}

1 Nous continuons ici la recension des thèses et mémoires d'archéologie ou de disciplines connexes soutenues dans l'Ouest ainsi que celle des travaux concernant à un titre ou à un autre l'archéologie du "Grand-Ouest» français, selon la formule initiée dans les précédents volumes de la RAO.

2 Compte tenu de la difficulté matérielle à obtenir un dépouillement exhaustif en la matière, la rédaction de la $R A O$ remercie chaleureusement par avance celles et ceux (notamment les lauréats et leurs directeurs de recherche) qui voudront bien lui faire part des références susceptibles de figurer utilement dans cette chronique, quel que soit le cadre - français ou étranger - dans lequel ces travaux auront été conduits et, par là même, contribuer à les faire connaître.

\section{Université de Rennes 1 - Beaulieu}

\section{Mémoires de Master 1}

Dreano, Maïwenn - Contribution à la connaissance du choix des matières premières mises en œuvre à Menez-Dregan 1 : Lithologie et morphométrie de l'outillage «lourd " de la couche 4. (dir. J.-L. Monnier, Archéo UR2).

4 Ravon, Anne-Lyse - Contribution à la connaissance du Paléolithique inférieur en Bretagne: l'exemple de la couche $4 \mathrm{du}$ site de Menez-Dregan 1, analyse technotypologique des nucleus. (dir. J.-L. Monnier, Archéo UR2).

\section{Mémoires de Master 2}

5 Beigl, Caroline (2009-2010) - Mise en place de méthodes d'analyse isotopiques sur la matière organique d'une tourbière pour une reconstitution paléoenvironnementale : contribution à l'archéologie. (dir. N. Marcoux, Archéo UR1). 

fonction des céramiques à usage culinaire : l'exemple de Port-Blanc (Hoëdic, Morbihan). (dir. M.-Y. Daire et R. March, Archéo UR2). l'arc atlantique des époques protohistorique et gallo-romaine. Étude des fibres et pigments. (dir. C. Brun, Université de Nantes ; J. Gomez de Soto et R. March, Archéo UR1). Tourneresse » (Calvados) et Argentan « Le Grand-Beaulieu » (Orne) : caractérisation et comparaison de deux corpus du Néolithique moyen bas-Normand. (dir. E. Ghesquière et J.-N. Guyodo, Archéo UR1).

11 Merdy, Élise (2009-2010) - Archéologie du bâti et origine des matériaux : du nouveau pour la chapelle Saint-étienne de Guer (56). (dir. G. Querré et J.-L. Monnier, Archéo UR1).

12 Mougne, Caroline (2009-2010) - Étude archéozoologique d'un site côtier du Second Âge du Fer: Dossen Rouz (Locquémeau-Trédrez, Côtes-d'Armor). (dir. C. Dupont et A. Baudry, Archéo UR1).

13 Papon, Justine (2009-2010) - L'enceinte de fossés interrompus de Champ-Durand (Nieulsur-L'Autize, Vendée): restitution des chaînes opératoires dans les contextes Néolithique récent et final. (dir. G. Marchand, Archéo UR1).

14 Pioffet, Hélène - Compléments d'enquête sur la périodisation des productions céramiques des îles anglo-normandes (Néolithique ancien et début du Néolithique moyen). (dir. L. Laporte et C. Marcigny, Archéo UR1).

Schaeffer, Erik (2009-2010) - Application du projet ALERT sur le littoral morbihannais et comparaison avec les autres programmes européens. (dir. M.-Y. Daire, Archéo UR1).

16 Taraud, Teddy - Les dépôts métalliques de Belle-Île-en-Mer (Morbihan, France) : étude topographique, typologique et technologique de trois ensembles datés du Bronze final 3 atlantique. (dir. S. Boulud-Gazo, MCF, Université de Nantes, et J. Gomez de Soto, CNRS, UMR 6566, Rennes).

17 Werthe, Élise (2009-2010) - Projet d'études dendrochronologiques de forêts subfossiles dans le quart nord-ouest de la France depuis 6000 ans. (dir. V. Bernard, Archéo UR1).

\section{Thèses de doctorat}

Lemouland, Quentin (2009) - Phytoarchéologie : impact des occupations archéologiques sur la flore actuelle (historique, concepts, méthodes et études de cas). (dir. J.L. Monnier et D. Marguerie, UMR 6566, Rennes).

19 Naudinot, Nicolas (2010) - Les dynamiques techno-culturelles et de peuplement au Tardiglaciaire dans le Grand-Ouest de la France. (dir. J.-L. Monnier, UMR 6566, Rennes) 


\section{Université de Rennes 1}

\section{Thèses de doctorat}

Kerdivel, Gwenolé (2009) - Occupation de l'espace et gestion des ressources à l'interface entre massifs primaires et bassins secondaires et tertiaires: l'exemple du Massif armoricain et de ses marges au Néolithique. (dir. J.-L. Monnier et L. Laporte, UMR 6566, Rennes).

Le Noac'h, Charlotte (2009) - La Poterie onctueuse en Bretagne, 10e-16e siècles. Étude archéologique, archéométrique et documentaire. Production, consommation, diffusion. (dir. B. Merdrignac, Université de Rennes 2, et J.-L. Monnier, Université de Rennes 1).

\section{Universités de Rennes 1 et Rennes 2}

\section{Mémoires de Master 1}

Cousseau, Florian - Des élévations dans les premières architectures monumentales en Europe occidentale. (dir. Luc Laporte, CNRS, UMR 6566).

\section{Université de Rennes 2}

\section{Mémoires de Master 1}

Amalric, Marion (2009) - Les châteaux du XIIIe siècle en Bretagne confrontés au modèle dit "philippien ». (dir. P.-Y. Laffont).

Baetens, Alix (2009) - Les pratiques funéraires à la fin du Moyen Âge dans les Pays de la Loire (Maine-et-Loire, Loire-Atlantique), 2 vol. (dir. P.-Y. Laffont).

Bracci, Simone - Le site de Bibracte et l'enclos PC 14 : le point sur les recherches et l'étude d'un ensemble de mobilier. (dir. J. Gomez de Soto, CNRS, et D. Vitali, université de Bologne).

Chebrou, Arnaud (2010) - La céramique de stockage protohistorique en Plaine de Caen. (dir. C. Marcigny, UMR 6566, avec la coll. de P. Giraud).

Edme, Laura - Les lingots et les déchets de fonderie dans les dépôts de l'horizon de l'épée à pointe en langue de carpe. (dir. C. Le Carlier de Veslud, CNRS, et M. Fily, UMR 6566).

Godard, Céline (2010) - L'habitat circulaire en Bretagne et Normandie à l'âge du Fer: un héritage britannique? (dir. C. Marcigny, UMR 6566).

Godet, Émilie (2009) - Le mobilier métallique civil et domestique issu de la zone 13 du château $d u$ Guildo, 2 vol. (dir. P.-Y. Laffont).

Grosset, Marie (2009) - Le Prieuré de Saint-Magloire de Léhon (Côtes-d'Armor), 2 vol. (dir. P.Y. Laffont).

Lamerant, Gabrielle - Les sépultures en coffre de pierre du Massif armoricain. Du néolithique au Bronze moyen : inventaire et bilan de la recherche. (dir. J.-N. Guyodo, MCF, Université de Nantes).

Lavoue, Maëva - Étude de la composition du mobilier métallique et première estimation des quantités de matières métalliques consommées (cuivre, étain, plomb) à l'âge du Bronze (du 
Bronze moyen I au Bronze final III) en Bretagne et Loire-Atlantique. (dir. C. Le Carlier de Veslud et M. Fily, UMR 6566).

\section{Université de Paris 1 (Panthéon - Sorbonne)}

\section{Mémoires de Master 1}

Le Goaziou, Edern (2010) - Le sel à l'âge du Bronze dans l'Europe du Nord-Ouest. (dir. C. Marcigny).

Marais, Axel (2009) - Les fortifications de Guérande, 2 vol. (dir. P.-Y. Laffont).

Nordez, Marilou - Bracelets de l'âge du Bronze et du Premier âge du Fer provenant de LoireAtlantique et du Maine-et-Loire. (dir. S. Boulud, MCF, Université de Nantes, et J. Gomez de Soto, CNRS, UMR 6566).

Perrin, Marie-Xuan - L'Âge du Fer en Écosse à travers les nouvelles découvertes aux détecteurs de métaux: objets de type indigène et romain. (dir. J. Gomez de Soto, CNRS, et I. Ralston, université d'Édimbourg).

Reinbold, Aurélie (2009) - Le paysage dans le nord-est rennais à l'époque médiévale. (dir. D. Pichot).

Remy, Julie - Les fortifications de l'âge du Fer en Pays de la Loire. Inventaire raisonné. (dir. M. Monteil, MCF, Université de Nantes, et J.-P. Bouvet, SRA, Pays de la Loire).

\section{Mémoires de Master 2}

Boeckler, Philippe (2009) - Champtoceaux (Maine-et-Loire). Étude archéologique d'un site de hauteur fortifié sur la longue durée, 2 vol. (dir. P.-Y. Laffont). de la zone 13. (dir. L. Beuchet et P.-Y. Laffont).

Godet, Émilie (2010) - Le mobilier métallique civil et domestique issu des communs et de la cuisine du château du Guildo (Côtes-d'Armor), 2 vol. (dir. P.-Y. Laffont).

Jeanneret, Lucie (2009) - L'habitat aristocratique fortifié et fossoyé dans une région de marche: les baronnies de Fougères et de Dol-Combourg face à la Normandie (Xe-XIIIe s.), 3 vol. (dir. P.-Y. Laffont).

Lagarrigue, Paul (2009) - Les édifices de culte chrétien bâtis au sein de structures galloromaines en milieu rural dans le nord-ouest de la Gaule, aux IVe-VIIIe siècles, 2 vol. (dir. P.Y. Laffont et C. Petit-Aupert).

Marais, Axel - Les fortifications de Guérande (Loire-Atlantique), 2 vol. (dir. P.-Y. Laffont).

Sitâ, André (2010) - Les manoirs périurbains dans l'ouest de la France. Le domaine de La Touche à Nantes, 2 vol. (dir. P.-Y. Laffont).

Tisserand, Guillaume (2010) - État des lieux historiographique, bibliographique et cartographique des fortifications de terre médiévales en Bretagne, 4 vol. (dir. P.-Y. Laffont).

Dupart, Olivia - Haches polies en silex dans le département de l'Eure: caractérisation et provenance. Étude des collections du Musée de l'Évêché à Évreux. (dir. F. Giligny).

Denis, Jan - Les céramiques à dégraissant végétal en Basse-Normandie: étude des pâtes, identification des végétaux et protocole expérimental. (dir. F. Giligny et X. Savary). 


\section{Mémoires de Master 2}

49 Bosset, Gabrielle (2009) - Hoëdic et sa nécropole mésolithique: "archéologie» d'une collection. Première étape d'un réexamen « anthropologique ». (dir. de B. Valentin). 